\title{
Exploring Local Perspectives for Conservation Planning: A Case Study from a Remote Forest Community in Indonesian Papua
}

\author{
Miriam van Heist ${ }^{1, *}$, Nining Liswanti ${ }^{2}$, Manuel Boissière ${ }^{2,3}$, Michael Padmanaba ${ }^{2,4}$, \\ Imam Basuki ${ }^{2,5}$ and Douglas Sheil ${ }^{1,2}$
}

1 Department of Ecology and Natural Resource Management (NMBU-INA), Norwegian University of Life Sciences, P.O. Box 5003, 1432 Ås, Norway; E-Mail: Douglas.Sheil@nmbu.no

2 Center for International Forestry Research (CIFOR), P.O. Box 0113 BOCBD, Bogor 16000, Indonesia; E-Mails: n.liswanti@cgiar.org (N.L.); m.boissiere@cgiar.org (M.B.); m.padmanaba@cgiar.org (M.P.); i.basuki@cgiar.org (I.B.)

3 Centre de Coopération Internationale en Recherche Agronomique pour le Développement (CIRAD), Campus de Baillarguet, Avenue Agropolis, 34398 Montpellier Cedex 05, France

4 Center for Integrative Conservation, Xishuangbanna Tropical Botanical Garden/Chinese Academy of Sciences (XTBG/CAS), Menglun, Yunnan 666303, China

5 Fisheries and Wildlife Department, Oregon State University, Corvallis, OR 97331, USA

* Author to whom correspondence should be addressed; E-Mail: Miriam.vanheist@nmbu.no; Tel.: +47-4861-9266.

Academic Editor: Eric J. Jokela

Received: 1 June 2015 / Accepted: 31 August 2015 / Published: 22 September 2015

\begin{abstract}
Reconciling conservation and livelihoods is a concern wherever forests are important in local people's lives. We plead for engaging these people in survey activities to clarify what is important to them, as a first step in conservation planning. This will help to address their priorities and gain their guidance and support for interventions. This paper presents the results of such a survey with the community of Kwerba in Mamberamo, a remote and little known part of Indonesian Papua. Views and priorities were explored through interviews, scoring exercises, community mapping and a field survey. Whereas small gardens provided most staple food, culture and livelihoods were linked to the forest. People scored primary forest highest for nearly all use categories. Primary forest was particularly highly valued as a source of construction materials, ornaments and rituals, and as a hunting place. We developed a list of the overall most important plants and animals. Many natural
\end{abstract}


resources were used, but few were commercially exploited. The community had rules to control access to certain areas and resources. Taboos to restrict access to sacred places were also maintained. Our evaluation identified opportunities to achieve conservation outcomes jointly with the Kwerba people. In follow-up activities, the community presented local government with a land-use plan for their territory. The government recognized the value of our approach and requested training to implement it more widely in the region.

Keywords: forest conservation; forest-dependent people; participatory approach; community mapping; scoring exercise; resource management; land use planning

\section{Introduction}

How to safeguard our world's biodiversity is among the greatest challenges of our age. Escalating demands for food, water and raw materials place ever increasing pressures on remaining natural habitats with the resulting trade-offs offering few easy solutions for conservation [1]. Various remedies have been suggested. For some the answer is expansion of strictly protected areas and increased exclusion of people [2]. Many advocate for massively increased conservation spending [3] and large-scale designation of remaining wilderness areas as conservation areas [4]. It remains unclear how realistic, ethical and sustainable such options are [5,6].

Protected areas already cover over $12 \%$ of the world's surface [7] with the greatest proportion in the tropics [8]. Although their expansion over recent years was dramatic, there are limits to what can be achieved by this approach alone. Even well-funded protected areas remain vulnerable to external threats [9-11]. In addition, there are mounting ethical and practical concerns whenever people are displaced by conservation [12] and numerous accounts of local stakeholders' hostility to conservation authorities as a result of exclusion have been noted [5,13]. Communities can become frustrated at seeing resources they once used and controlled being poorly protected when they themselves would intervene if they were authorized to do so [14].

At the same time as the concern about global biodiversity loss has grown, appreciation of the role that local people play in determining habitats and maintaining species has also increased [15] as has concern about loss of human cultures and languages [16]. Many have highlighted the need to reconcile local people's needs and culture with global conservation goals [17-19] and to offer a more ethical and democratically accountable form of conservation [6,20].

While there is considerable support for such a more locally led conservation, there is also widespread skepticism [21,22]. In the past, conservation planning has generally been top-down and areas gained a designated status that restricted future use. To help modify this planning culture we developed a series of simple methods and guiding principles to work with local people from the onset, to help outsiders identify relevant attitudes and information and find opportunities to build on these [23-27]. This approach appears particularly promising in regions where conservation authorities are weak or absent, and global conservation organizations have had little influence thus far. Ideally the local authorities and other local stakeholders would see the value of clarifying opportunities for conservation and engage with the process in a manner that can be scaled up over large regions. 
In this paper we describe the application of such an approach in the Mamberamo-Foja watershed in the Indonesian province of Papua - this densely forested area is one of the world's least studied regions and contains biodiversity of global significance [28-34]. Human population density is low, with people concentrated in small, scattered settlements. Livelihoods depend on wild resources and small scale farming. Until recently, problems of access, information, disease (malaria), and insecurity have discouraged large scale deforestation [23,33,34]. However, this is changing with (coal) mining, timber extraction, plantation developments and a dam threatening the region's forests [32-34].

We wanted to document local perspectives and values regarding the landscape and the traditional use and management of natural resources. Additional questions surrounded the willingness of the communities to engage and participate in this process, and whether the local government, and other communities in the region might be persuaded of the utility of our approach. Given the limited information available on Mamberamo and the potential for achieving conservation in this poorly known region we provide an overview of our results and experiences with one community. We discuss briefly how communities may be supported to maintain their forests and natural resources, in view of external pressures and the recent rapid changes taking place around them.

\section{Methods}

In Sheil et al. [24] we present a full description of the methods. We worked with a multidisciplinary team of eight, primarily local university students and government staff.

\subsection{Study Area}

Our survey took part in the Mamberamo-Foja watershed of West Papua. Conservation International (CI) started developing a conservation support program for this area in 2000 and had had initial discussions with a few communities. Our team was invited to identify and explore the opportunities for conservation with these communities and carried out our surveys in July 2004. Here we present the one with the people of Kwerba (Figure 1). At the time, Kwerba was not yet officially recognized as a village; since 2007 it has been under the administration of the new Mamberamo Raya Regency. The village is accessible from Jayapura-Papua's capital—by small aircraft, from the regency capital Kasonaweja and other villages by boat.

Less than a kilometer from the settlement, closed canopy forest begins and continues to the Foja Mountains. In 1982, the mountains and surrounding lands were officially designated as a Wildlife Reserve (Ministry of Agriculture decree No. 782/Kpts/Um/10/1982) which the Ministry of Forestry extended down to the coast in 1999 (No. 820/Kpts/Um/11/82). Since then the Mamberamo-Foja Wildlife Reserve has spanned around 20,000 $\mathrm{km}^{2}$ and includes a range of altitudes from sea level to $2100 \mathrm{~m}$ elevation (Figure 1). Kwerba's territory lies inside this reserve. Legally any extractive activity is prohibited, but the protected area authority (Balai Besar Konservasi Sumber Daya Alam or Natural Resources Conservation Agency) has no presence in the regency to enforce regulations. 


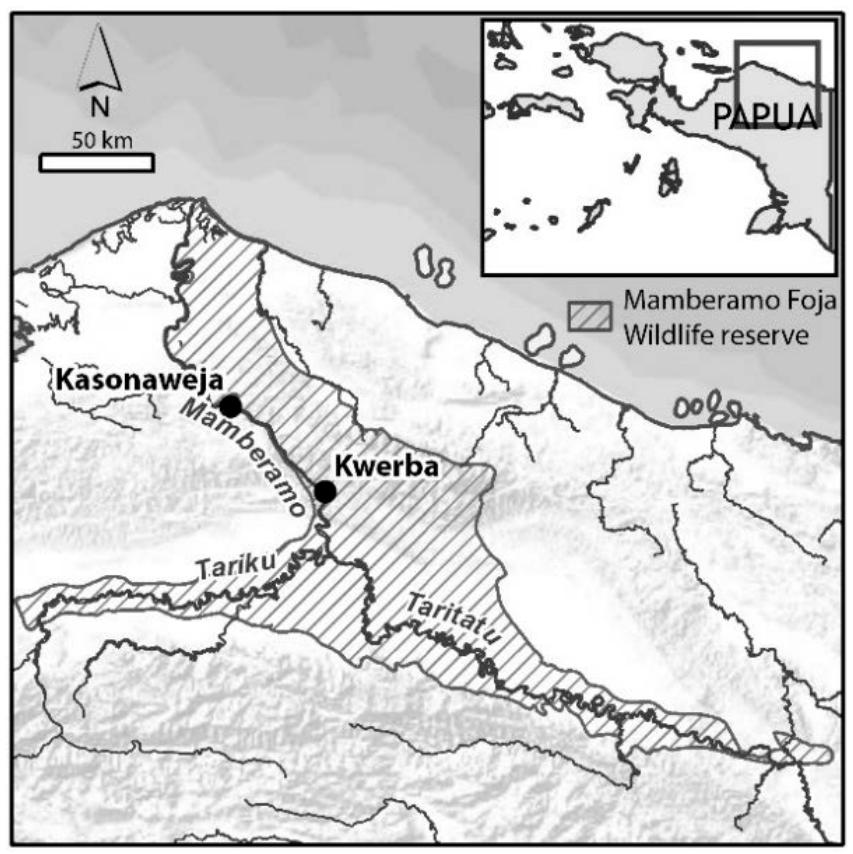

Figure 1. Location of the study area in Papua.

Various stakeholders (Mamberamo Raya Regency departments, Conservation International, community leaders) started discussing a change in status from wildlife reserve to national park. A park status might allow the distinction of a core conservation zone and one where some extractive use of resources by communities is allowed. This would offer local people the possibility of negotiating resource utilization agreements and joint forest management.

Meanwhile, local government programs focus on economic development and income from the region's resources.

\subsection{Community Meetings, Interviews and Household Survey}

Two months prior to the survey, two team members visited Kwerba to explain the objectives of the upcoming activities and to ask the customary leader and his community for permission and participation. Soon after arrival of the survey team (July 2004), we held a community meeting. Once more, we presented our objectives, answered questions and asked people's participation, while introducing our team. In the following days, team members interviewed eight key informants including the village head and customary and religious leaders. These interviews covered history (of settlement, land use and important events), traditional leadership, cultural practices, and rules and regulations with regard to resource management.

We conducted a survey with household heads, and recorded demographic information (gender, age, religion, education, occupation and household composition), sources of livelihood, individual land tenure, what people perceive as threats to the forest (and their response), and their aspirations for the future. We covered 32 of the 44 households, determined by availability of the household heads during our stay. 
We used Indonesian, the national language taught at schools, for all communications with community members. Sometimes a village youth assisted with translating to and from Kwerba while we talked to people who were unable to speak Indonesian.

\subsection{Participatory Mapping}

On the second day, we started a participatory mapping exercise to let the Kwerba people introduce us to their landscape. Formal maps were non-existent, and we had copied the Mamberamo River and major tributaries, as well as the location of Kwerba and the mountain ridge from a 2001 satellite image (Landsat path 102, row 062). Large sheets with this basic topographic information were then used as base maps (scale 1:75,000) for the mapping exercise.

We formed four groups of three to six people, older men and younger men, older women and younger women, respectively, all "knowledgeable” villagers as proposed by community members (an "older" person would generally have grandchildren, and be older than 35). These four groups worked separately, on different base maps. People initially oriented themselves, added names to the tributaries and drew additional streams. We then asked them to list important landscape features (hills, lakes, swamps) and land types (gardens, different forest types), specific resources (certain animals, wood, sago, medicines) and "special sites" and to mark their locations. Usually clan members filled in names and resources in their own territories. The groups met three or four times for one to four hours each. In the process, our team built a common terminology and understanding of the land with community members. We publicly displayed the maps during our stay and villagers would often examine them and discuss issues amongst themselves or with us.

Guided by these preliminary maps, our field team visited all key land and forest types, accompanied by two clan members of the respective areas present some results of this biophysical part of the survey elsewhere [35]. We also gathered GPS coordinates from key points during the field survey, which allowed us to georeference the base map and improve the resulting maps. We assisted community members in combining all information from the four respective maps onto one. The community permitted us to (hand) make another copy, for our reference.

\subsection{Scoring Exercises}

We used the "Pebble Distribution Method" (PDM) [24,36,37] to let people score the importance of locally valued land and forest types, use categories as well as resources, already identified in conjunction with the mapping activities. Again, the community selected people to participate in the scoring exercises and we formed four groups, consisting of four to six younger and older, men and women respectively (these were not necessarily the same people as those who had participated in the mapping). In total 20 people participated in the scoring exercises.

Our facilitator showed participants cards with a drawing plus the local name of land and forest types. She then asked the groups to distribute 100 "pebbles" (beans in our case) over the cards to represent how important they found these for specific use categories: she explained that twice as many beans on A than on B meant "for that use A is twice as important to us as B". Once the group agreed on the scores, the facilitator recorded them and asked for an explanation, especially for zero scores or ones that were 
higher/lower than expected. Any new insights or miscommunications were followed up with further questions [36].

Here we present the results from three scoring exercises:

(1) the relative importance of land types, for each use category,

(2) the relative importance of forest types for each use category, and

(3) the relative importance of the ten "most important" species listed for each use category (plants and animals separately). This exercise includes a pre-score of the relative importance of each use category.

For exercises (1) and (2), we calculated “combined scores” for each PDM by adding the four individual group scores for a specific category and dividing the total by four.

In exercise (3), people often considered more than 10 species important and we asked them to give an additional score for "all those not mentioned", called "the rest-score".

We calculated the Local User Value Index (LUVI) for each species (see Sheil et al. [24] for details of the calculation). The LUVI normalizes the scores by the rest-score for that category and takes into account the relative importance of the use category itself. This allows adding the LUVIs of a species that had scored in more than one use category, to arrive at "overall importance” for each species. The higher the overall LUVI score, the more important the species was to local people. The total LUVI score of each PDM group was 100.

We present the top 10 plants and animals in the two most important use categories food and heavy construction. We also ranked all species and present a list of the 10 most important plants and animals to the people of Kwerba.

\section{Results}

We have divided the results in three sections by first presenting contextual information about the community and its livelihood, followed by the importance of land types and resources, and finally the traditional management of territory and resources.

\subsection{Description of Context}

\subsubsection{Settlement and Population}

The Kwerba settlement is located on a small plateau surrounded by hills, an hour on foot from the mouth of the Wiri, a tributary of Mamberamo River. People settled here in 1974.

In 2004 the population was 354 people, and 44 households. Five clans were recognized as territory owners; the Tawane (19 households), Meop (8 households), Maner (4 households), Haciwa (4 households) and Karawata ( 1 household). Five more clans ( 8 households) were present as they had married into Kwerba from outside.

There were 21 of 44 household heads with no formal education. Of the 23 others, 15 had only finished elementary school, four completed junior high school and four senior high school. The main self-reported occupations were "farmer" (28) and "hunter" (12), two household heads considered themselves teachers, another two were a trader and a priest. All were Christians (Protestant). The village had a church building 
that doubled as an elementary school. Official medical services were absent but there was a paramedic in the village.

\subsubsection{Livelihoods; Main Sources of Food and Income}

The following information was obtained from interviews with key informants. Some aspects we confirmed with the household survey and our own observations.

Sago was the staple food. People had planted sago palms (Metroxylon sagu Rottb.), but also obtained sago from wild stands. Furthermore, they planted mixtures of sweet potatoes (Ipomoea batatas (L.) Lam.), yams (Dioscorea spp.), cassava (Manihot esculenta Crantz), pumpkin (Cucurbita maxima Duchesne) and fruit trees (banana (Musa spp.), papaya (Carica papaya L.), coconut (Cocos nucifera L.), cocoa (Theobroma cacao L.) and red pandanus (Pandanus conoideus Lam.)), in small gardens near the settlement. Larger forest trees were often still present in the gardens.

Kwerba's territory contained a network of rivers, swamps and lakes. Informants told us they caught and ate fish on a daily basis, and especially various catfish (family Ariidae) were readily available. They also hunted, for their own consumption and occasionally for cash. Hunting two to eight hours was usually sufficient to catch enough meat to feed a family for one or two days. People stated that animals were still abundant around Kwerba and that the availability of meat had changed little since they settled. We also noted that juvenile kangaroos, cassowaries and wild piglets were kept as pets.

Trading crocodile skins had been an important way to earn cash. Usually groups of two to four men who could afford fuel and other supplies would hunt crocodiles. They salted the skins for preservation. Since 2000, a company (PT. Bintang Mas, Jayapura, Indonesia) regularly visited the village to buy the skins, but hunters also directly sold them to traders in Kasonaweja and Jayapura. Informants reported various by-products of the skin trade: meat, eggs, genitals (eaten to "make men stronger"), fat (used as massage oil), teeth (to make needles and rings) and bones (to make arrowheads), all still valuable for own use and trading.

Other marketable products included the dried swim bladders from catfish (ikan sembilang: Arius sp., a non-native species introduced by migrants), sold to traders in Chinese traditional medicine; birds of paradise (in particular Paradisaea minor and Seleucidis melanoleucus), hunted for feathers and the live bird trade; resin from Agathis spp. (particularly A. alba), valued locally for lighting (burned in lamps); the fragrant eaglewood (or gaharu, from fungus-infected stems of Aquilaria spp.), sold for incense trade; and birds' nests, produced by cave swifts (Aerodramus fuciphagus and A. maximus) and highly valued for soup by Chinese traders (collection was only sporadic, though, as access to the remote caves was difficult and costly).

\subsubsection{Aspirations and Threats to Livelihoods}

All household heads agreed that life had improved since Kwerba received government recognition and village development assistance. However, they were still awaiting a functioning school, church and community health center. Many stated their desire for access to roads, services (clean water, power) and markets, but also expressed fear that improved accessibility might lead to logging and mining companies coming to the regency, making them more vulnerable to outside exploitation of their resources. Through contacts with downstream villages, the Kwerba community was aware of ongoing logging and coal and 
gold exploration elsewhere and had learned of other villages' experiences with investors (in neighboring Burmeso, for example, the timber company PT. Mamberamo Alas Mandiri obtained a forest concession and the community now had restricted access to the forest). All respondents wished for official recognition of their land ownership. They worried they may otherwise lose control of their forest.

They also recognized other threats to the environment and their livelihoods and mentioned specifically poison-fishing and overexploitation (particularly of crocodiles, catfish and birds of paradise).

\subsection{Scoring the Importance of Land Types, Forest Types and Species}

\subsubsection{The Importance of Land Types}

People scored forest as most important for 12 of 14 use categories ((Table 1): See a brief description of all categories in Table 2). Particularly high were these scores in the boat building category, for basketry/cordage, for hunting tools and for ornament/ritual. Young women even scored forest as the only place of value for the latter three categories. Interestingly people gave the highest scores to gardens — not forest - for two of the most important categories, food and firewood.

For the food category, all land types received a score. The settlement, gardens and sago plantations scored a total of 48 3/4, whereas river and lakes scored $19 \frac{1}{2}$. People explained that the low forest score (13 1/4) was because most daily food requirements were met by the nearby gardens and sago, as well as the river. Note that forests scored 33 for hunting, and other land types were valued too. River and lakes had a total score of $44 \frac{1}{4}$, considerably higher than forest.

Forest scored 33 3/4 for heavy construction. People pointed out that their houses did not require much timber but were constructed from poles and sago fronds, available near the settlement.

For firewood, people most valued their gardens. These were close to their homes, and typically contained a mix of wild and planted trees. People took dead branches or selectively cut trees for firewood. We questioned the high score for rivers in the firewood category and were told that abundant good wood floated down the Mamberamo River and was deposited on the shore near the village.

Table 1. The importance of land types for each respective use category. Use categories are ordered from highest to lowest importance score and described below. Grey highlighted and bold are the highest scoring land types for each category, lighter grey shows the second highest score. All data presented are combined scores of four PDM groups.

\begin{tabular}{|c|c|c|c|c|c|c|c|c|c|c|c|}
\hline $\begin{array}{l}\text { Use Category } \\
\text { (Overall Importance Score) }\end{array}$ & 䒕 & 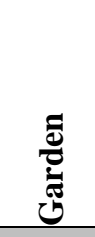 & 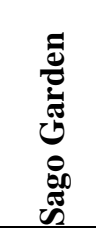 & 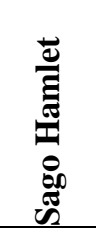 & 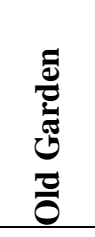 & 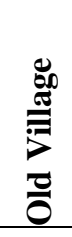 & 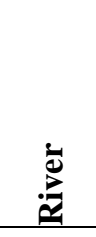 & 突 & 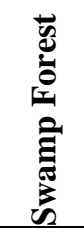 & $\begin{array}{l}\bar{D} \\
\dot{0} \\
\dot{0} \\
\end{array}$ & 흉 \\
\hline Food $(12$ 1/4) & $103 / 4$ & 15 & $11_{1 / 2}^{1 / 2}$ & $11 \frac{1}{2}$ & $43 / 4$ & $63 / 4$ & $101 / 4$ & $91 / 4$ & 7 & $13^{1 / 4}$ & 100 \\
\hline Heavy construction (12 1/4) & 9 & 3 & $101 / 4$ & 16 & $15^{1 / 4}$ & $31 / 4$ & $31 / 4$ & $23 / 4$ & $31 / 2$ & $333 / 4$ & 100 \\
\hline Firewood (10 1/4) & $21 / 2$ & $251 / 4$ & 5 & 5 & $11 \frac{1 / 2}{2}$ & $33 / 4$ & $191 / 4$ & $4 \frac{1}{2}$ & $21 / 2$ & $203 / 4$ & 100 \\
\hline Hunting place (8 1/4) & $1 / 2$ & $11 / 4$ & $41 / 4$ & $63 / 4$ & $33 / 4$ & 4 & $243 / 4$ & $19 \frac{1}{2}$ & $21 / 4$ & 33 & 100 \\
\hline Boat building (7 1/2) & $31 / 2$ & $43 / 4$ & $1 / 4$ & $1 / 2$ & $41 / 2$ & $3 / 4$ & $181 / 4$ & $151 / 2$ & $31 / 2$ & $481 / 2$ & 100 \\
\hline Tools (7) & 6 & $43 / 4$ & $93 / 4$ & 11 & $41 / 2$ & 1 & $161 / 4$ & 7 & $31 / 4$ & $361 / 2$ & 100 \\
\hline
\end{tabular}


Table 1. Cont.

\begin{tabular}{|c|c|c|c|c|c|c|c|c|c|c|c|}
\hline $\begin{array}{l}\text { Use Category } \\
\text { (Overall Importance Score) }\end{array}$ & 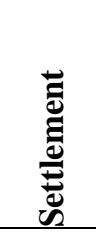 & 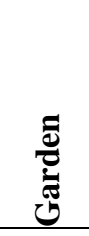 & 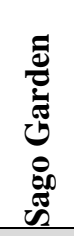 & 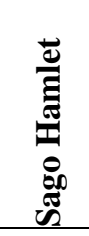 & 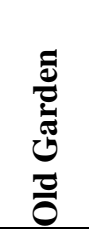 & 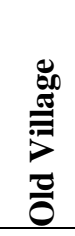 & 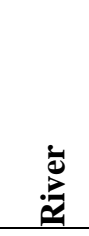 & 菣 & 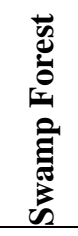 & 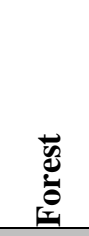 & हैं \\
\hline Future (7) & $13^{1 / 4}$ & $71 / 4$ & 14 & $133 / 4$ & $43 / 4$ & $4^{1 / 4}$ & $93 / 4$ & $73 / 4$ & $4 \frac{1}{1} 2$ & $203 / 4$ & 100 \\
\hline Medicine (6 1⁄2) & 14 & $103 / 4$ & 5 & $53 / 4$ & 7 & $53 / 4$ & $11 \frac{1 / 4}{4}$ & $10 \frac{1}{2}$ & $61 / 4$ & $233 / 4$ & 100 \\
\hline Basketry/Cordage (6 1/2) & $11 / 2$ & $23 / 4$ & $21 / 4$ & 4 & $73 / 4$ & $4 \frac{1}{4}$ & $81 / 4$ & $91 / 2$ & $61 / 4$ & $53 \frac{1}{1 / 2}$ & 100 \\
\hline Marketable items (6) & 10 & 10 & 11 & $10 \frac{1 / 2}{2}$ & $2 \frac{1}{4}$ & 3 & $143 / 4$ & $15^{1 / 2}$ & $31 / 2$ & $19 \frac{1 / 2}{2}$ & 100 \\
\hline Hunting tools (5 3/4) & $71 / 2$ & $61 / 4$ & $23 / 4$ & $23 / 4$ & $53 / 4$ & $9 \frac{1 / 2}{2}$ & $83 / 4$ & $13 / 4$ & $31 / 2$ & $51 \frac{1}{1 / 2}$ & 100 \\
\hline Light construction (4 1/4) & 5 & $61 / 4$ & 5 & $13^{1 / 4}$ & $19^{1 / 4}$ & $61 / 2$ & 5 & 4 & $4^{1 / 2}$ & $31 \frac{1}{4}$ & 100 \\
\hline Ornament/Ritual (3 3/4) & $81 / 2$ & 0 & $2 \frac{1}{1 / 2}$ & $91 / 4$ & $63 / 4$ & 4 & 4 & $4 \frac{1 / 4}{4}$ & $23 / 4$ & 58 & 100 \\
\hline Recreation (2 3/4) & $24 \frac{1}{2}$ & $10^{1 / 2}$ & 0 & 0 & 0 & 0 & 22 & $61 / 2$ & 0 & $361 / 2$ & 100 \\
\hline
\end{tabular}

Table 2. Description of Use Categories and Land Types.

\begin{tabular}{|c|c|}
\hline Use Categories & Description \\
\hline Food & Primary and secondary foods \\
\hline Heavy construction & Timber for house construction and bridges \\
\hline Firewood & Fuel for cooking, warmth \\
\hline Hunting place & Indirect, benefiting hunting (usually when fruiting) \\
\hline Boat building & Timber for making boats (not oars or poles) \\
\hline Tools & Tools in agriculture, boating, household use \\
\hline Future & General (not explained in detail) \\
\hline Medicine & Medicinal and health related \\
\hline Basketry/Cordage & For weaving or tying \\
\hline Marketable items & Products sold for cash \\
\hline Hunting tools & Spear handle, bait, poison, trap \\
\hline Light construction & For making temporary shelter, chicken or pig runs, fences \\
\hline Ornament/Ritual & Used in ceremony, dress, jewellery and decoration \\
\hline Recreation & For entertainment, rest \\
\hline \multicolumn{2}{|l|}{ Land Types } \\
\hline Settlement & Human habitation; semi permanent houses, church, school \\
\hline Garden & Area planted with a mix of annual and perennial species \\
\hline Sago garden & Area planted with sago palms \\
\hline Sago hamlet & This term includes wild stands as well as planted but not maintained sago gardens \\
\hline Old garden & Abandoned garden, where forest has regenerated \\
\hline Old village & Abandoned village (before 1974). Planted fruit trees and sago remained, as well as graves \\
\hline River & Mamberamo and its tributaries \\
\hline Lake & Small waterbodies, scattered around Kwerba \\
\hline Swamp forest & Area of wet, (seasonally) flooded forest \\
\hline Forest & Primary, non-swamp forest \\
\hline
\end{tabular}

\subsubsection{The Importance of Forest Types}

We compared the scoring results for different forest types (Table 3) and found that primary forest received the highest score for all use categories except firewood. People said that primary forest provided 
timber (boat building scored particularly high) and was the best place for hunting, and for finding ornament/ritual materials. We also noted its high score for the future category, as a reserve of resources as well as of fertile land for crop growing. Secondary forests (often located near the settlement) were particularly important for firewood, medicine and food (fruits, wild or from earlier planted fruit trees). The low score for swamp and Agathis forests, despite their valuable resources, was due to the distance and inaccessibility, meaning they were seldom visited.

Table 3. The importance of different forest types for each respective use category. Combined scores of four PDM groups. Grey highlighted and bold are the highest scoring forest types for each category, lighter grey shows the second highest score.

\begin{tabular}{|c|c|c|c|c|c|c|c|}
\hline $\begin{array}{l}\text { Use Category } \\
\text { (Overall Importance Score) }\end{array}$ & 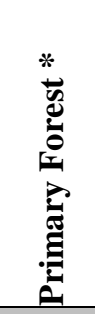 & 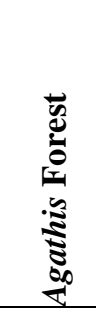 & 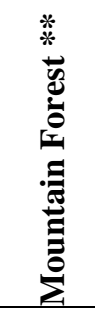 & 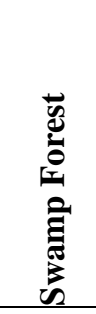 & 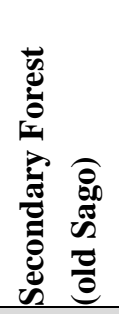 & 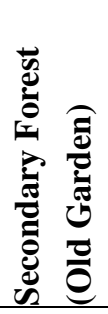 & 즁 \\
\hline Food (12 1/4) & $281 / 4$ & $101 / 2$ & $123 / 4$ & 10 & $241 / 2$ & 14 & 100 \\
\hline Heavy construction (12 1/4) & 37 & 0 & $91 / 4$ & 11 & $291 / 4$ & $131 / 2$ & 100 \\
\hline Firewood (10 1/4) & $301 / 2$ & $51 / 2$ & $123 / 4$ & 5 & $61 / 2$ & $393 / 4$ & 100 \\
\hline Hunting place (8 1/4) & $501 / 2$ & $71 / 2$ & 17 & 8 & $91 / 4$ & $73 / 4$ & 100 \\
\hline Boat building ( $71 / 2)$ & $641 / 2$ & $81 / 4$ & 15 & $61 / 2$ & $1 / 2$ & $51 / 4$ & 100 \\
\hline Tools (7) & $43 \frac{1}{1 / 2}$ & $101 / 2$ & $161 / 4$ & $71 / 2$ & $81 / 2$ & $133 / 4$ & 100 \\
\hline Future (7) & 27 & $201 / 4$ & 18 & $10^{1 / 2}$ & $93 / 4$ & $14_{1 / 2}$ & 100 \\
\hline Medicine (6 1/2) & $351 / 4$ & 10 & $16^{1 / 2}$ & $81 / 2$ & $143 / 4$ & 15 & 100 \\
\hline Basketry/Cordage (6 1⁄2) & $431 / 2$ & 15 & $123 / 4$ & 7 & $93 / 4$ & 12 & 100 \\
\hline Marketable items (6) & $46 \frac{1}{4}$ & $7 \frac{1}{2}$ & 22 & $81 / 4$ & $103 / 4$ & $51 / 4$ & 100 \\
\hline Hunting tools (5 3/4) & 433 & 7 & 13 3/4 & 8 & $83 / 4$ & 18 3/4 & 100 \\
\hline Light construction (4 1/4) & $401 / 4$ & $83 / 4$ & 21 & $51 / 4$ & $91 / 2$ & $15^{1 / 4}$ & 100 \\
\hline Ornament/Ritual (3 3/4) & $533 / 4$ & $63 / 4$ & $171 / 4$ & $71 / 2$ & $4 \frac{1 / 2}{2}$ & $10^{1 / 4}$ & 100 \\
\hline Recreation (2 3/4) & $513 / 4$ & 8 & 20 & $61 / 2$ & 6 & $73 / 4$ & 100 \\
\hline
\end{tabular}

See notes under Table 1 for description of use categories; * Primary forest (hutan rimba) refers to lowland forest here; ** Mountain forest (hutan gunung); Agathis forest (also in the mountains) was distinguished by itself.

\subsubsection{The Most Important Species}

We combined the LUVIs of the four scoring groups (the total score was thus 400). The combined "rest-group" of unnamed species in the various categories amounted to 146.31 (37\% of the total). The named species- 157 plants and 64 animals-therefore accounted for $63 \%$ of the total value. In Table 4 , we list the 10 top scoring species of plants and animals, and present the use categories in which they each scored. 
Table 4. The overall most important species of plants (a) and animals (b).

\begin{tabular}{|c|c|c|c|c|c|c|c|c|c|c|c|c|c|c|c|c|c|}
\hline & & & & $\mathbf{a}$ & & & & & & & & & & & & & \\
\hline $\begin{array}{l}\text { TOP } 10 \text { Plants } \\
\text { Local Name (s) }\end{array}$ & 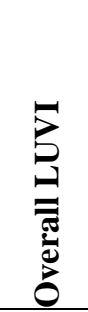 & $\begin{array}{l}\text { (Total Number of Species Scored = 157), } \\
\text { Species Name; Indonesian; English }\end{array}$ & $\underset{8}{8}$ & 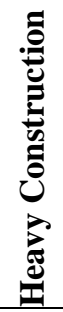 & 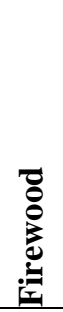 & 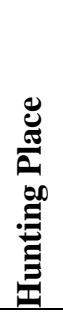 & 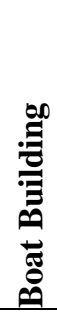 & $\stackrel{\infty}{8}$ & 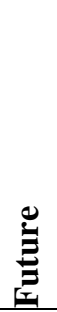 & : & 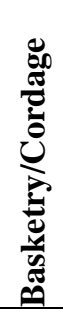 & 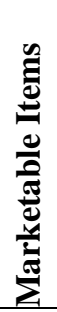 & 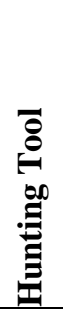 & 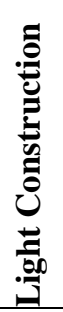 & 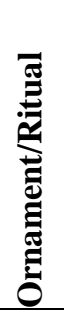 & 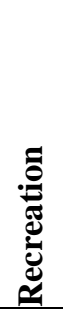 & 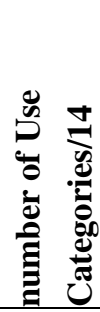 \\
\hline $\begin{array}{l}\text { Kuikar/kwekar/ } \\
\text { kwikar }\end{array}$ & 9.69 & Calophyllum sp.; kayu bitangur & $\mathrm{x}$ & $\mathrm{X}$ & $\mathrm{x}$ & & $\mathrm{X}$ & & $\mathrm{X}$ & & & $\mathrm{x}$ & $\mathrm{x}$ & $\mathrm{x}$ & & $\mathrm{x}$ & 9 \\
\hline Wac & 7.93 & $\begin{array}{l}\text { Gnetum gnemon L.; melinjo; gnetum/ paddy } \\
\text { oats }\end{array}$ & $\mathrm{X}$ & $\mathrm{x}$ & $\mathrm{x}$ & & & $\mathrm{x}$ & & & $\mathrm{X}$ & $\mathrm{X}$ & $\mathrm{x}$ & & $\mathrm{x}$ & & 8 \\
\hline $\begin{array}{l}\text { Panipiroric/ } \\
\text { panibidoric }\end{array}$ & 6.41 & Ficus sp.; kayu goro; fig tree & & & & & $\mathrm{X}$ & $\mathrm{X}$ & $\mathrm{x}$ & & & $\mathrm{X}$ & & & & & 4 \\
\hline Cirih & 5.55 & $\begin{array}{l}\text { Intsia bijuga (Colebr.) Kuntze; kayu merbau; } \\
\text { ironwood/ipil }\end{array}$ & & $\mathrm{X}$ & & $\mathrm{x}$ & & & $\mathrm{X}$ & & & & $\mathrm{X}$ & & & & 4 \\
\hline (Kaniw) namuk & 5.26 & Metroxylon sagu Rottb.; sago; true sago palm & $\mathrm{X}$ & $\mathrm{x}$ & & $\mathrm{x}$ & & & $\mathrm{X}$ & $\mathrm{x}$ & & $\mathrm{X}$ & $\mathrm{x}$ & & $\mathrm{X}$ & & 8 \\
\hline Hiber/hiper & 5.14 & $\begin{array}{l}\text { Pometia pinnata J.R. Forst. \& G. Forst.; } \\
\text { matoa; island lychee }\end{array}$ & $\mathrm{X}$ & & $\mathrm{x}$ & $\mathrm{X}$ & $\mathrm{x}$ & $\mathrm{x}$ & $\mathrm{x}$ & & & $\mathrm{x}$ & $\mathrm{x}$ & $\mathrm{x}$ & & & 9 \\
\hline $\begin{array}{l}\text { Mampai/ } \\
\text { Nampai }\end{array}$ & 5.08 & Unidentified; kayu merah & & & & & $\mathrm{X}$ & $\mathrm{x}$ & $\mathrm{x}$ & & & $\mathrm{x}$ & & & & & 4 \\
\hline Haruk & 4.64 & Unidentified; kayu seman & & $\mathrm{X}$ & & & & & & & $\mathrm{x}$ & & $\mathrm{x}$ & $\mathrm{X}$ & & & 4 \\
\hline Piric & 3.30 & Gulubia costata (Весс.) Весс. & & $\mathrm{x}$ & & $\mathrm{x}$ & & $\mathrm{X}$ & $\mathrm{x}$ & & $\mathrm{x}$ & & & & & & 5 \\
\hline Petanic & 3.07 & Unidentified (fruit eaten by pigs) & & $\mathrm{x}$ & & $\mathrm{x}$ & & & & & $\mathrm{x}$ & & $\mathrm{x}$ & & & & 4 \\
\hline \multicolumn{3}{|c|}{ Number of top 10 species in this use category } & 4 & 7 & 3 & 5 & 4 & 5 & 7 & 1 & 4 & 6 & 7 & 3 & 2 & 1 & \\
\hline
\end{tabular}


Table 4. Cont.

\begin{tabular}{|c|c|c|c|c|c|c|c|c|c|c|c|c|c|c|c|c|c|}
\hline & & & & b & & & & & & & & & & & & & \\
\hline $\begin{array}{l}\text { TOP } 10 \text { Animals } \\
\text { Local Name (s) }\end{array}$ & $\begin{array}{l}\text { 忿 } \\
\text { 是 } \\
\overline{0} \\
0 \\
0\end{array}$ & $\begin{array}{l}\text { (Total Number of Species Scored = 64), } \\
\text { Species Name; } \\
\text { Indonesian; English * }\end{array}$ & $\underset{\substack{0\\
}}{D}$ & 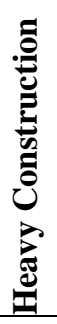 & 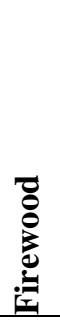 & 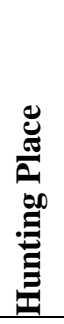 & 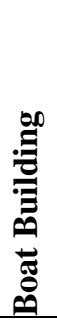 & $\stackrel{n}{\stackrel{0}{0}}$ & $\underset{\Xi}{\Xi}$ & 刍 & 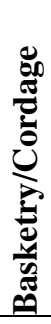 & 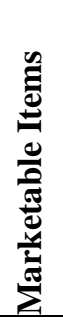 & 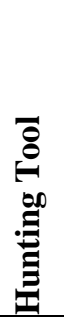 & 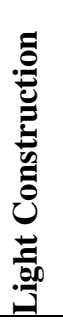 & 氖 & 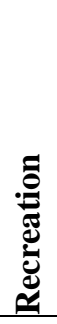 & 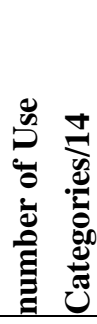 \\
\hline Kama (h) & 9.08 & $\begin{array}{l}\text { Casuarius unappendiculatus; kasuari; northern } \\
\text { cassowary VU }\end{array}$ & $\mathrm{X}$ & & & & & $\mathrm{X}$ & $\mathrm{X}$ & $\mathrm{x}$ & $\mathrm{x}$ & $\mathrm{X}$ & $\mathrm{X}$ & & $\mathrm{X}$ & $\mathrm{x}$ & 9 \\
\hline Cidam/ciram & 7.10 & $\begin{array}{l}\text { Crocodylus novaeguineae; buaya irian; New } \\
\text { Guinea crocodile LC }\end{array}$ & $\mathrm{x}$ & & & & & $\mathrm{x}$ & $X$ & $\mathrm{X}$ & & $\mathrm{X}$ & $\mathrm{x}$ & & & & 6 \\
\hline Cipic & 6.74 & Sus scrofa; babi hutan; wild pig/boar LC & $\mathrm{X}$ & & & & & $\mathrm{X}$ & $\mathrm{x}$ & $\mathrm{X}$ & & $\mathrm{X}$ & & & $\mathrm{x}$ & $\mathrm{x}$ & 7 \\
\hline Hower & 5.37 & $\begin{array}{l}\text { Dorcopsis hageni; lao-lao; greater forest } \\
\text { wallaby LC }\end{array}$ & $\mathrm{x}$ & & & & & $\mathrm{X}$ & $\mathrm{x}$ & & & $\mathrm{x}$ & $\mathrm{X}$ & & & $\mathrm{X}$ & 6 \\
\hline Kaci (h/j) & 4.26 & $\begin{array}{l}\text { Paradisaea minor; cendrawasih kecil; lesser } \\
\text { bird-of-paradise LC }\end{array}$ & & & & & & & $\mathrm{X}$ & & $\mathrm{x}$ & $\mathrm{X}$ & & & $\mathrm{X}$ & $\mathrm{X}$ & 5 \\
\hline Habwa & 3.75 & $\begin{array}{l}\text { Dendrolagus inustus; kanguru; grizzled tree } \\
\text { kangaroo VU }\end{array}$ & $\mathrm{X}$ & & & & & $\mathrm{x}$ & $\mathrm{x}$ & $\mathrm{x}$ & & $\mathrm{x}$ & $\mathrm{x}$ & & $\mathrm{x}$ & $\mathrm{x}$ & 8 \\
\hline Maris & 3.67 & $\begin{array}{l}\text { Goura victoria; mambruk Victoria; Victoria } \\
\text { crowned pigeon NT }\end{array}$ & $\mathrm{x}$ & & & & & $\mathrm{x}$ & $\mathrm{x}$ & & & $\mathrm{x}$ & $\mathrm{X}$ & & $\mathrm{x}$ & $\mathrm{x}$ & 7 \\
\hline Pawir & 3.66 & $\begin{array}{l}\text { Cacatua galerita; kakatua putih; sulfur-crested } \\
\text { cockatoo LC }\end{array}$ & & & & $X$ & & & $\mathrm{X}$ & & & $X$ & $\mathrm{x}$ & & $\mathrm{x}$ & $\mathrm{X}$ & 6 \\
\hline Koreta & 2.36 & $\begin{array}{l}\text { Megapodius sp.; maleo/ayam hutan; } \\
\text { megapode/scrubfowl }\end{array}$ & $\mathrm{X}$ & & & $\mathrm{x}$ & & $\mathrm{x}$ & $\mathrm{x}$ & & & $\mathrm{X}$ & & & & & 5 \\
\hline Kahuk & 2.06 & $\begin{array}{l}\text { Murexechinus melanurus; tikus air; black-tailed } \\
\text { dasyure LC }\end{array}$ & $\mathrm{x}$ & & & & & $\mathrm{X}$ & & & & & & & & & 2 \\
\hline Number of top 10 & ies in $t$ & is use category & 8 & 0 & 0 & 2 & 0 & 8 & 9 & 4 & 2 & 9 & 6 & 0 & 6 & 7 & \\
\hline
\end{tabular}

$\mathrm{X}=$ species ranking high in the category; $\mathrm{x}=$ scored, but lower ranking. * IUCN status: VU = Vulnerable, NT = Near Threatened, LC=Least Concern. LUVI: the Local User Value Index. 
The plant with the highest LUVI (9.69) was a tree, Calophyllum sp. All 10 top species had timber value and scored for construction, boat building or both (like Calophyllum).

Plant species ranking high for food were taking second, fifth and sixth position in the overall plant top 10; the fruits and seeds, young leaves and flowers of Gnetum gnemon L. are eaten, sago from the Metroxylon sagu Rottb. palm is a staple and the fruits of Pometia pinnata J.R. Forst. \& G. Forst. were scored equally as food and as important for hunting place. Animals are attracted by the fruits too and when in season, hunting near these trees becomes easy.

The top ranking animals were particularly valued as food, marketable items, tools and future. People gave the highest rank to the cassowary (LUVI 9.08), valued for eight other uses than food and scoring high in six of them. Crocodiles and wild pigs came second (7.10) and third (6.74). The remaining species included marsupials such as a wallaby, kangaroo and dasyure but also birds like the lesser bird of paradise and sulphur-crested cockatoo, both valued as marketable items, and the Victoria crowned pigeon and Megapode, valued as food. People considered nine of the top ranking species important for the future.

All but one (the animal kahuk) of the twenty top species had scored in at least four use categories. Four plants and two animals even scored in eight or more. Table 5 presents the top species in the two most important use categories: food and heavy construction. Among food plants tamih (Artocarpus sp., breadfruit) had the highest LUVI (2.28). Tamih only scored as food, and both its fruits and seeds are eaten. However, in the medicine category, a plant called tantamih was mentioned-as a tea against cough and colds — and is possibly a part of tamih. Gnetum, Pometia and Metroxylon sagu Rottb. appeared all in the top five. Two species of wild yam were listed. The leaves of two listed fern species in the genus Nephrolepsis are eaten as vegetable, as are two other unidentified plant species.

Table 5. The most important species (plants and animals respectively) for food and for heavy construction (plants only).

\begin{tabular}{lcll}
\hline FOOD PLANTS & & & \\
\hline Local Name (s) & LUVI & Species Name; Indonesian; English & Notes \\
\hline & & (Total Number of Species Scored = 20) & \\
\hline Tamih & 2.28 & Artocarpus sp.; sukun hutan; wild breadfruit & fruits and seeds \\
Wac & 2.00 & Gnetum gnemon L.; melinjo; gnetum/paddy oats & nut, strobilus and leaves \\
Papar & 1.19 & Dioscorea sp.1; umbi hutan; a wild yam (1) & tubers \\
& & Pometia pinnata J.R. Forst. \& G. Forst.; matoa; island & fruits \\
Hiper & 1.15 & lychee & staple food; stem pith \\
& 1.00 & Metroxylon sagu Rottb.; sago; true sago palm & leaves \\
Kaniw) namuk & 0.98 & Unidentified & tubers \\
Kwarah & 0.96 & Dioscorea sp.2; umbi hutan; a wild yam (2) & young red leaves \\
Harut & 0.80 & Nephrolepis sp.1; paku-pakuan; fern (1) & young green leaves \\
Kanar & 0.79 & Nephrolepis sp.2; paku-pakuan; fern (2) & leaves \\
Pakiu & 0.77 & Unidentified & \\
\hline
\end{tabular}


Table 5. Cont.

\begin{tabular}{|c|c|c|c|}
\hline \multicolumn{4}{|l|}{ FOOD ANIMALS } \\
\hline Local Name (s) & LUVI & Species Name; Indonesian; English & IUCN Status \\
\hline & & (Total Number of Species Scored $=20$ ) & \\
\hline Cipic & 1.45 & Sus scrofa; babi hutan; wild pig/boar & LC \\
\hline Kama (h) & 1.10 & $\begin{array}{l}\text { Casuarius unappendiculatus; kasuari; northern } \\
\text { cassowary }\end{array}$ & VU \\
\hline Habwa & 1.09 & Dendrolagus inustus; kanguru; grizzled tree kangaroo & VU \\
\hline Koreta & 1.03 & $\begin{array}{l}\text { Megapodius sp.; maleo/ayam hutan; } \\
\text { megapode/scrubfowl }\end{array}$ & \\
\hline Hower/lao-lao & 0.96 & Dorcopsis hageni; lao-lao; greater forest wallaby & LC \\
\hline Maris & 0.80 & $\begin{array}{l}\text { Goura victoria; mambruk Victoria; } \\
\text { Victoria crowned pigeon }\end{array}$ & NT \\
\hline Karah & 0.65 & $\begin{array}{l}\text { Dendrolagus sp.; kanguru; golden-mantled? } \\
\text { tree kangaroo }\end{array}$ & \\
\hline Asian & 0.57 & Peroryctes raffrayana; tikus tanah; Raffray‘s bandicoot & LC \\
\hline Kapahen & 0.44 & Varanus sp; biawak; monitor lizards & \\
\hline Kahuk & 0.39 & $\begin{array}{l}\text { Murexechinus melanurus; tikus air; } \\
\text { black-tailed dasyure }\end{array}$ & LC \\
\hline \multicolumn{4}{|c|}{ HEAVY CONSTRUCTION } \\
\hline Local Name (s) & LUVI & Species Name & Notes \\
\hline & & (Total Number of Species Scored $=20)$ & \\
\hline Cirih & 3.61 & $\begin{array}{l}\text { Intsia bijuga (Colebr.) Kuntze; kayu merbau; } \\
\text { ironwood/ipil }\end{array}$ & $\begin{array}{l}\text { Used as poles and } \\
\text { beams, also tables }\end{array}$ \\
\hline Kuikar/kwekar/kwikar & 2.45 & Calophyllum sp.; kayu bitangur & $\begin{array}{l}\text { Used as poles or for } \\
\text { floors }\end{array}$ \\
\hline Haruk & 2.38 & Unidentified; kayu seman & $\begin{array}{l}\text { Used for roofs (bark for } \\
\text { floors) }\end{array}$ \\
\hline Namuk & 1.88 & Metroxylon sagu Rottb.; sago; true sago palm & $\begin{array}{l}\text { Woven leaves as roof } \\
\text { cover, bark as walls }\end{array}$ \\
\hline Piric & 1.85 & Gulubia costata (Весc.) Becc. & Used for floors \\
\hline Petanic & 1.74 & Unidentified & Used for walls \\
\hline Kanawar & 1.42 & Unidentified & $\begin{array}{l}\text { Used for floors (and } \\
\text { leaves for roofs) }\end{array}$ \\
\hline Micebwa & 1.41 & Unidentified & Used for floors or walls \\
\hline Namotac & 1.41 & Unidentified & $\begin{array}{l}\text { Leaves used as roof } \\
\text { cover }\end{array}$ \\
\hline Kwih & 0.93 & Unidentified & Used for floors \\
\hline
\end{tabular}

People gave the wild pig the highest LUVI (1.45) among animals for food. A second species of tree kangaroo (possibly golden mantled), the Raffray's bandicoot and a monitor lizard appear in the list, as well.

The five most important species for heavy construction (houses, bridges) have all been mentioned already as overall top 10 species. Intsia bijuga (Colebr.) Kuntze topped with a LUVI of 3.61. The other five were non-identified species. 


\subsection{Traditional Management of Territories and Natural Resources}

\subsubsection{Mapping of Territories, Sites and Resources}

We learned from the mapping exercise that Kwerba claimed a traditional territory of $1100 \mathrm{~km}^{2}$. Each of the five clans claimed an area coinciding with the catchment of one or more Mamberamo tributaries (Figure 2a).

Many people showed extensive and detailed knowledge of their clan's territories. Men contributed most of the information about upstream areas, sacred places, hunting areas, valuable timber trees and reserved areas. Women mostly gave information related to crops, gardens and resources near the settlement.

Sago stands (wild and cultivated) were extensive. The largest "garden” areas (mixtures of bananas, cassava and vegetables) were located near the settlement, in Maner territory. Villagers had also established banana and cassava gardens along the river of their own clan's territory, to have a source of food when hunting or patrolling.

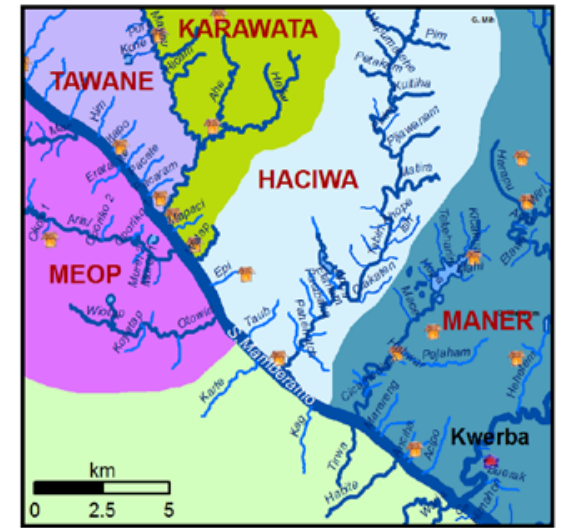

(a) The respective clan areas

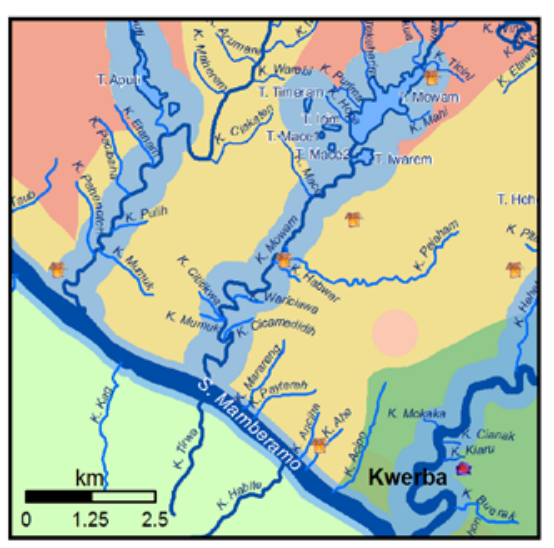

(c) Land use

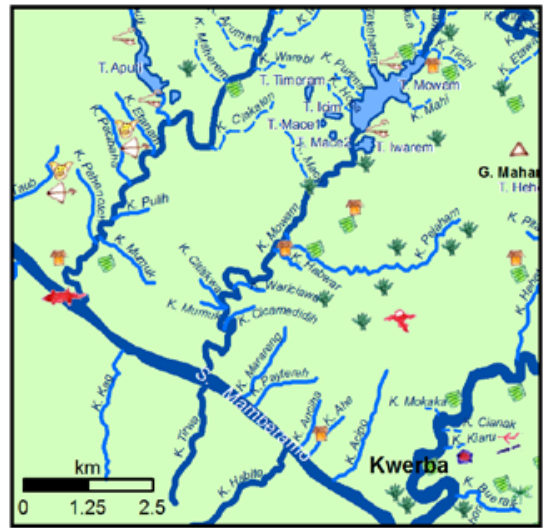

(b) Natural resources

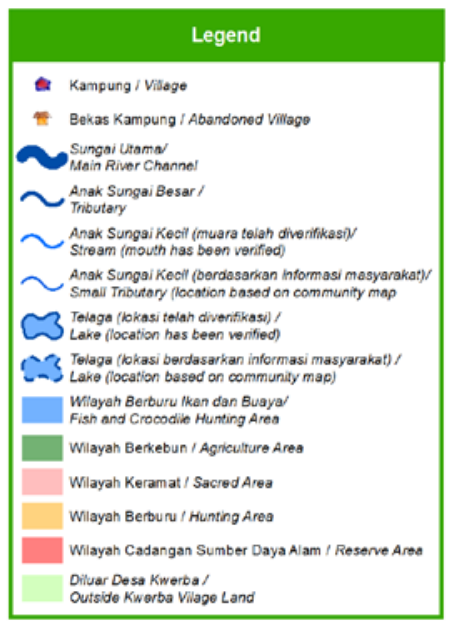

Figure 2. Sections of the participatory map and a common legend. (a) The respective clan areas; (b) Natural resources; (c) Land use.

Good locations marked for specific resources included locations for fishing and hunting crocodiles (lakes and rivers such as the Tabiri and Mowam), hunting pigs and cassowaries, collecting resin, eaglewood and birds' nests (Figure 2b. The legend does not explain the symbols, as requested by the community). 
People indicated "Special sites" on the map which included salt springs, natural pools and hot springs. Salt springs were visited regularly by wild pig and cassowary and hence were good hunting places. People valued pools and hot springs as places to simply visit and relax. Some of the special sites were considered "sacred" (see below).

At a more generalized level, four important "land use zones” (see Figure 2c) were distinguished:

(1) Cultivation zone. Close to the principal settlement, in Maner territory near the Wiri River.

(2) Hunting zones. Extensive forest areas located along each clan's main tributary.

(3) Reserved zones. Upstream of the tributaries, in primary forest.

(4) Sacred areas. Scattered over the territory.

The rules and practices associated with these areas are further detailed below.

\subsubsection{Area Protection}

Key informants told us that the boundaries between Kwerba and neighboring villages had been agreed among the respective traditional leaders. In the mapping exercise they showed how Kwerba's territory itself was divided over its five clans (see Figure 2a) and explained that each clan protected its own area from unauthorized exploitation. In general, if outsiders wanted to enter the Kwerba territory, they first needed permission from the customary leader. If sites or resources in a specific clan's territory were sought, that clan leader would have to give his permission as well. Outsiders had to abide by local regulations and were never allowed to cut trees. We learned from the customary leader of a case where Kasonaweja people put fish poison in the river within Kwerba territory. However, only a warning was given and no fine applied. In another case, construction workers from Gorontalo, who were building in Kwerba, were fined three million IDR (around 250 US\$) when they cut trees down for their own use [38].

Each of the clan leaders might allow individuals from other Kwerba clans to cultivate or hunt within his territory, as long as it was done for their own consumption. In particular, all villagers preferred cultivating close to the settlement in Maner territory and thus had to ask the Maner leader for permission. Villagers also had to request permission for collecting plants in the territories of other clans, but such requests were usually granted. Cutting forest for timber or swidden cultivation was restricted to clan members.

Patrols of the clan area by boat or on foot were carried out each month for one or two days. Alternatively, a guard stayed at the mouth of the tributary to check people on return. If rules were broken, the catch would be confiscated, and the clan leader would decide whether a warning or additional sanction was warranted.

Additional customary forest management systems were still in place. For example, the Kwerba community had allocated a large extent of mountain forest "more than one day walking from the village" as reserved area, a place for preserving plants and animals. General harvesting of resources in the reserved area was restricted. In specific circumstances, however, e.g., during monitoring of the territory, people were allowed to hunt or cut a sago tree for sustenance.

The customary leader assessed the state of important natural resources based on his own observations and those of other clan members and evoked or adapted regulations accordingly. He could declare a Teter, which meant that an area was (temporarily or permanently) closed for collection or hunting of a specified resource. Such had been the case for crocodiles in Kwerba (see below). A Teter also existed 
for two species of birds of paradise (Paradisaea minor and Seleucidis melanoleucus), in a "small area, dominated by Dipterocarp trees, which used to be frequented by the birds" [38]. We could not verify this in the field.

People recognized "sacred sites or areas" in their territory. These included hot springs, rocks in rivers as well as the Foja Mountains. People believed that their ancestors inhabit these places in the form of plants and animals and that they should not be disturbed. All sacred areas were protected by traditional law and considered off-limit for everyone without express permission from the customary leader. Before entering any sacred site, a ritual had to be performed.

Taboos restrained the use of certain plants and animals, too. Villagers believed that adhering to the taboos averted bad luck. We learned of no sanctions for breaking a taboo, but adherence appeared to be general and unquestioned.

The customary leader could demand a fine from someone violating any rules. These procedures were respected at the time of our survey. Leaders explained that customary regulations - though never written-were well known by to the community and any changes were shared verbally.

\subsubsection{Protection or Restoration of Specific Resources}

\section{Crocodiles}

In the 1980s, an FAO (Food and Agriculture Organization of the United Nations) project had assessed the crocodile population in Mamberamo and suggested to hunt only those of 28-56 cm "commercial belly width”, to protect the (larger-sized) adult breeding population and also to restrict egg collection to a few specified lakes $[39,40]$. Initially, the community had rejected the FAO recommendations, regarding them as external interference. At that time, a hunter could still collect 5-10 crocodiles in one overnight hunt. However, late in the 1980s, even one crocodile per trip was not always achieved. Overexploitation by a company developing a crocodile farm, the Kwerba community itself as well as neighboring villages had drastically reduced the crocodile population.

The customary leader of Kwerba had then imposed the FAO's suggested size restrictions on crocodile hunting and declared a Teter. He set a quota for the number of animals that could be caught and required that hunters should seek a permit from him. In addition, specific lakes and rivers were closed to crocodile hunting for several years and the collecting of eggs was regulated. Compliance had been good and crocodile numbers had increased [41]). At the time of our survey, villagers monitored local crocodile populations, both where hunting was still allowed as well as where it was prohibited, to judge the need for extending, maintaining or ending the Teter.

\section{Catfish}

During interviews, we were told that "outsiders" had used tuba, a poison extracted from the root of Derris elliptica (Fabaceae), to catch catfish in the Mamberamo River and that the catfish population had seriously declined. The customary leader had subsequently banned the use of fish poison. If someone was found using tuba, a warning would first be given, but if this was ignored, the catch would be confiscated and the leader might set a fine. 
Birds of Paradise

Birds of paradise were normally hunted in the mountain and swamp forest, where they displayed on trees such as ironwood (Intsia bijuga (Colebr.) Kuntze), Ficus sp. and ketapang (Terminalia sp.). Two species, Paradisaea minor and Seleucidis melanoleucus used to be common in Kwerba. Due to a decline in some areas, the customary leader limited hunting to 12 tributaries where the birds were still abundant. This rule applied from 2000 onwards and the customary leader said that villagers complied [38].

\section{Specific Plant Resources}

Plant species protected by customary rules included ironwood (Intsia bijuga), matoa (Pometia pinnata J.R. Forst. \& G. Forst.), gaharu (Aquilaria sp.) and orchids. Selling these had provided local people with additional income, but had led to concerns of overexploitation. At the time of the survey, clan members were only allowed to cut ironwood and matoa for personal use. Some areas were closed for gaharu collecting for several years and alternative areas had been allocated.

\section{Discussion}

Gaining knowledge of remote communities poses challenges [42], but we are confident that our survey provided a good summary of local perspectives. Our understanding resulted from four weeks of living and working in the village while we engaged with the majority of adults. Language may have restricted expression, but informants generally appeared at ease whether sharing their views with us directly or through an interpreter.

We set out to explore and identify opportunities for developing a shared conservation vision with a remote forest community in Papua and documented the following insights:

- People depended on natural resources for their livelihoods.

- For 12 of 14 use categories people found forests the most important land type.

- For food supply, however, people most valued their gardens, sago palms and the river.

- The most important species were Calophyllum sp., cassowary (Casuarius unappendiculatus), gnetum (Gnetum gnemon L.), crocodile (Crocodylus novaeguineae) and wild pig (Sus scrofa).

- People still respected customary leadership.

- Customary control systems and regulations for protecting sites and resources were operational and were updated when necessary.

- People recognized "reserved areas," where activities were regulated, and "sacred areas," where all access was restricted.

- People saw exploitation by outsiders as a threat, but wanted to benefit from development, without losing control over their lands, forest and resources.

We developed mutual trust and a shared understanding about the traditional ways to manage and protect the forest, which provided a basis for discussing conservation and other land management options in follow-up activities. The community members were keen to engage with and participate in our survey and we noted that people often kept debating issues amongst themselves after our joint exercises.

This community thus does not appear to be a threat to forest biodiversity and its effective conservation, as is sometimes said about local communities in general $[43,44]$. Population density is very low, and the 
Kwerba people are not primarily dependent on forest for food (though protein supply from hunting remains important). In fact, they have protected local biodiversity, as forest-dependent communities often do [45-49]. They are keen to maintain their access to forest resources and protect them from outsiders.

We documented the situation in 2004, admittedly a while ago, but some of the authors revisited the community several times between 2004 and 2012, and confirm that above attitudes have not changed.

A summary of follow-up activities follows, as we recognize the importance of a longer presence and commitment for our approach to have an impact (see [50] for a review of ten cases). Eight months after the initial surveys, we returned to Kwerba. We presented and invited feedback on the results. People were happy, particularly with the laminated map. They said that the map was a good documentation of the many natural resources and important places in their territory, and would be crucial for discussions and negotiations with others concerning developments that might impact Kwerba people's livelihoods. Another meeting was held to discuss aspirations for future land use and resource management. In 2010, we facilitated community members to draft a map with "proposed land use zones”. This closely reflected the map of existing land use (as in Figure 2c). People explained that they did not want any major changes within their territory, nor any road construction or plantations. If the Mamberamo-Foja Wildlife Reserve becomes a National Park, they want their use zones to be recognized and included.

The trust built during our close involvement with the Kwerba community was crucial for its leaders to allow a team of biologists to access the sacred Foja Mountains a year after the survey [23]. That survey gained international recognition for the area, as several new or long-lost species of animals and plants were discovered [51-54].

In 2007, the Kwerba community, assisted by Conservation International (CI), drafted a "Community Conservation Agreement” governing access to and use of natural resources by outsiders. This was a first attempt to formalize the traditional stewardship. Unfortunately CI discontinued working on such agreements and no further developments can be reported.

We had hoped to persuade local government and other communities in the region of the utility of the approach. Again, follow-up events partly confirm that: in 2012, Kwerba's leaders presented the maps, draft agreement and other results in a land use planning workshop for civil servants of the regency [55]. The regency government was impressed and responded by requesting training in participatory approaches for its civil servants, which took place a few months later. During the training, the approach was adapted for wider implementation. Also, the Mamberamo Raya Regency, the Papua provincial administration and the Indonesian Forest and Climate Support program signed an agreement for a region-wide project to protect the forest, while supporting economic development and improving food security [56]. This project will use surveys adapted from the one presented here.

The Indo-Pacific Conservation Alliance chose our approach to explore the views and priorities of local Asmat people in and around Lorentz National Park. This has resulted in negotiated joint management agreements between the park authorities and the communities [57].

\section{Looking Ahead}

West Papua is experiencing rapid economic and social developments. A timber company is already operating elsewhere in Mamberamo Raya, and coal and plantation companies are discussing 
opportunities with the regency government. Communities have also been approached, and feel increasingly vulnerable due to their lack of recognized land rights [38]. Their ability to judge proposals is undermined by their limited experience. Downstream Burmeso has experienced the effects of large-scale logging and road construction in the forest around the village. That forest is now degraded and hunting has become more difficult, but people also report the economic benefits of the operations [55].

The Kwerba community has heard of land use developments but has so far not experienced them. Some members complain that the conservation status of the Mamberamo-Foja reserve restrains their development options, but some others recognize that this currently protects them from other developments claiming control over their land. People would welcome improved access to health services, education, markets and the various trappings of development and a cash economy. They do call on the government to provide these benefits. Change is desirable and unavoidable. Our focus is not on preventing these developments and changes, but in ensuring that we can maximize the gains and minimize the losses to people and their environment. It is not a question of development OR conservation, but rather of how to achieve development along with the protection of land and resources that the people themselves find desirable. Population densities are low. In such circumstances it is possible to be optimistic that development can be achieved without undermining traditional values and livelihoods and without destroying the large tracts of unique and biodiverse forest that these communities wish to see maintained for future generations.

Land use and development planning in consultation with communities provide a starting point, and indicate that the regency is taking that obligation seriously [55]. Decentralization has led to Mamberamo Raya's government being less than one day's travel from most villages, facilitating communication. Another positive development is that the leader (bupati) of Mamberamo Raya was elected directly and people consider him "theirs,", and thus answerable to their concerns.

Sheil et al. [49] documented local systems of resource monitoring and control that contribute to maintaining conservation values in three other Mamberamo community territories. They highlight the people's wish to maintain a strong connection with the land, but also emphasize that these systems are not static but adapt as circumstances and opportunities change. Positive outcomes for conservation are therefore not guaranteed and will depend on context, options and motivations.

How could local people be supported to manage and protect their forests and other resources? Some answers are clear and well known. For example, Agrawal and Angelsen [58] derive lessons from successful community forest management projects and emphasize sufficient size and clear boundaries of forests, predictability of benefit flows, local autonomy in designing clear and enforceable rules for access and use of forests, and provisions for monitoring and sanctioning rule violations. They found that a stable technological and policy environment, low levels of intergroup conflict, and small to medium-sized, forest-dependent user groups that have management experience were the best contextual characteristics associated with successful forest outcomes (see also [59,60]). The FAO 2007 review of tenure in Southeast Asian forestry adds capacity-building opportunities for local people to a similar list of success factors [61].

To increase the likelihood of successful community protection of their land and forests the people of Kwerba (and of Mamberamo in general) would benefit from legal recognition of their ownership and rights over their traditional lands. Such rights should include community autonomy in regulating and monitoring forest access and use as well as sanctioning against violations, and clear agreements on who benefits from forest cover maintenance and income from natural resources (ensuring "predictability of 
benefit flows”). At the same time community members must gain the skills and knowledge required to recognize and respond to external threats, demands and opportunities. This will ensure that the communities can make well informed choices that benefit them and their future generations.

The Kwerba community was and is still a "small forest-dependent user group with management experience and low level of intergroup conflict in a relatively stable technological environment”, as mentioned in [58]. The policy environment is changing: Indonesia has an increasingly positive attitude towards recognizing local communities' rights over traditional territories. A milestone was the 2014 Constitutional Court ruling that traditional forest land (hutan adat) should no longer be considered state forest (hutan negara). While many details remain unclear, this ruling opens the door to granting legal rights to land based on established claims. Many local and international non-governmental organisations are supporting communities by explaining opportunities, preparing them for change and assisting in proposal writing. It seems likely that the success of any claims would be improved by evidence of historic forest use and good stewardship. A simple set of questions and exercises like the one presented here could potentially help inform and guide such a process, both for the authorities, and for the communities involved.

Various forms of outside support to communities exist and will remain important for some time to come. Building local capacity in mapping, resource assessments, land use planning and negotiations, legal rights, democracy and governance will allow communities to become more equal partners in discussions about their land. Also, facilitating community leaders to travel to places where developments they have never seen before have already taken place (e.g., coal mining on a large scale in Kalimantan or logging in Burmeso), to look around and to talk to all involved about the experience, would be a contribution to letting communities make informed choices on their own.

The Mamberamo-Foja area is a wildlife reserve under provincial government control. However, shortage of staff and resources for managing conservation areas have led to absence of practical management or control. The Mamberamo Raya regency would like to see the area being declared a national park, which status may allow zonation and co-management by communities like Kwerba. They are already looking after this vast forest area and would be happy to continue doing so, if certain conditions were met. Already existing reserved and sacred areas should be recognized and traditional control maintained or expanded. Access to locally important species and areas should be part of the negotiations. From a conservation perspective, special attention should be given to the sustainable management of the most valued animal species which are classified as Vulnerable (Northern Cassowary, Casuarius unappendiculatus, and Tree Kangaroos, Dendrolagus spp.) or Near Threatened (Crowned Pigeon, Goura victoria).

Collaboration must be the best way forward and exclusion on the other hand would likely lead to resentment $[23,49]$. A meta-analysis of peer reviewed articles on 40 protected areas and 33 community managed forests in Latin America, Asia and Africa also found that community managed forests presented lower and less variable annual deforestation rates than protected forests [62]. Also, the FAO review of forest tenure in Southeast Asia concluded that community forestry, build on traditional structures and with consideration of local values for resources, was more likely to be successful than forestry management that replaced these structures and values [61]. 


\section{Conclusions}

Recognizing the presence and role of local people, knowing what is important to them and involving them from the start are crucial for changing the culture of conservation planning. We have documented how people of a remote forest community in Papua relate to their environment. We characterized what is important to them and described their traditional resource management. In the process, the community discussed and clarified their concerns and aspirations amongst themselves and communicated these to us and to other outsiders including the Mamberamo Regency authorities. The authorities welcomed the outcome and requested training in participatory methods for land use assessment and planning.

Local people's nearness, motivation, knowledge and activities make them key partners in conservation programs. Developing such programs based on local preferences, needs and aspirations offers a more ethical and democratic means to achieve conservation outcomes, while maintaining local livelihoods. Conflicts and associated long-term costs will then be reduced and conservation programs more effective and sustainable.

\section{Acknowledgments}

We are grateful for the hospitality we received from the Kwerba people and the interest and patience with which they answered all our questions and participated in the exercises.

We would like to thank Neville Kemp, CI-Papua Program Manager, Yoseph Watopa, Hugo Yoteni, Tommy Wakum, Yafeth Watori and Yance Bemei for all support received. Many thanks to M. Agus Salim at CIFOR for preparing the maps for this paper.

This study was funded by the European Commission and The Gordon and Betty Moore Foundation, in collaboration with Conservation International (CI) Indonesia, Indonesian Institute of Sciences (LIPI), Natural Resources and Environment Management Agency (BPSDALH) Papua Province, and Natural Resources Conservation Agency (BKSDA) Papua Province.

\section{Author Contributions}

All authors took part in data collection. Miriam van Heist, Nining Liswanti and Douglas Sheil developed the article and finalized it, with input from all other authors.

\section{Conflicts of Interest}

The authors declare no conflict of interest.

\section{References}

1. Sayer, J.; Sunderland, T.; Ghazoul, J.; Pfund, J.L.; Sheil, D.; Meijaard, E.; Venter, M.; Boedhihartono, A.K.; Day, M.; Garcia, C.; et al. Ten principles for a landscape approach to reconciling agriculture, conservation, and other competing land uses. Proc. Natl. Acad. Sci. USA 2013, 110, 8349-8356.

2. Hutton, J.; Adams, W.M.; Murombedzi, J.C. Back to the barriers? Changing narratives in biodiversity conservation. Forum Dev. Stud. 2005, 32, 341-370. 
3. McCarthy, D.P.; Donald, P.F.; Scharlemann, J.P.; Buchanan, G.M.; Balmford, A.; Green, J.M.H.; Bennun, L.A.; Burgess, N.D.; Fishpool, L.D.C.; Garnett, S.T.; et al. Financial costs of meeting global biodiversity conservation targets: Current spending and unmet needs. Science 2012, 338, 946-949.

4. Mittermeier, R.; Mittermeier, C.; Brooks, T.; Pilgrim, J.; Konstant, W.; da Fonseca, G.A.; Kormos, C. Wilderness and biodiversity conservation. Proc. Natl. Acad. Sci. USA 2003, 100, 10309-10313.

5. Redpath, S.M.; Young, J.; Evely, A.; Adams, W.M.; Sutherland, W.J.; Whitehouse, A.; Amar, A.; Lambert, R.A.; Linnell, J.D.C.; Watt, A.; et al. Understanding and managing conservation conflicts. Trends Ecol. Evol. 2013, 28, 100-109.

6. Sheil, D.; Meijaard, E.; Angelsen, A.; Sayer, J.; Vanclay, J.K. Sharing future conservation costs. Science 2013, 339, 270-271.

7. Chape, S.; Harrison, J.; Spalding, M.; Lysenko, I. Measuring the extent and effectiveness of protected areas as an indicator for meeting global biodiversity targets. Philos. Trans. R. Soc. Ser. B: Biol. Sci. 2005, 360, 443-455.

8. Brooks, T.M.; Wright, S.J.; Sheil, D. Evaluating the success of conservation actions in safeguarding tropical forest biodiversity. Conserv. Biol. 2009, 23, 1448-1457.

9. Baker, J.; Milner-Gulland, E.; Leader-Williams, N. Park gazettement and integrated conservation and development as factors in community conflict at Bwindi Impenetrable Forest, Uganda. Conserv. Biol. 2012, 26, 160-170.

10. Scholte, P.; Degroot, W. From debate to insight: Three models of immigration to protected areas. Conser. Biol. 2010, 24, 630-632.

11. Solomon, J.; Jacobson, S.K.; Wald, K.D.; Gavin, M. Estimating illegal resource use at a Ugandan park with the randomized response technique. Hum. Dimens. Wildl. 2007, 12, 75-88.

12. Agrawal, A.; Redford, K. Conservation and displacement: An overview. Conserv. Soc. 2009, 7, 1-10.

13. Sharpe, B. "First the forest": Conservation, “community" and "participation" in south-west Cameroon. Africa 1998, 68, 25-45.

14. Boissière, M.; Sheil, D.; Basuki, I.; Wan, M.; Le, H. Can engaging local people’s interests reduce forest degradation in Central Vietnam? Biodivers. Conserv. 2009, 18, 2743-2757.

15. Ellis, E.C.; Ramankutty, N. Putting people in the map: Anthropogenic biomes of the world. Front. Ecol. Environ. 2008, 6, 439-447.

16. Gorenflo, L.J.; Romaine, S.; Mittermeier, R.A.; Walker-Painemilla, K. Co-occurrence of linguistic and biological diversity in biodiversity hotspots and high biodiversity wilderness areas. Proc. Natl. Acad. Sci. USA 2012, 109, 8032-8037.

17. Adams, W.M.; Hulme, D. If community conservation is the answer in Africa, what is the question? Oryx 2001, 35, 193-200.

18. Kaimowitz, D.; Sheil, D. Conserving what and for whom? Why conservation should help meet basic human needs in the tropics. Biotropica 2007, 39, 567-574.

19. Mcshane, T.O.; Hirsch, P.D.; Trung, T.C.; Songorwa, A.N.; Kinzig, A.; Monteferri, B.; Mutekanga, D.; Thang, H.V.; Dammert, J.L.; Pulgar-Vidal, M.; et al. Hard choices: Making trade-offs between biodiversity conservation and human well-being. Biol. Conserv. 2011, 144, 966972.

20. Vermeulen, S.; Sheil, D. Partnership for tropical conservation. Oryx 2007, 41, 434-40. 
21. Mavhunga, C. Even the rider and a horse are a partnership: A response to Vermeulen \& Sheil. Oryx 2007, 41, 441-442.

22. Robinson, J.G. Recognizing differences and establishing clear-eyed partnerships: A response to Vermeulen \& Sheil. Oryx 2007, 41, 443-444.

23. Sheil, D.; Boissière, M. Local people may be the best allies in conservation: Letter to the editor. Nature 2006, 440, 868-868.

24. Sheil, D.; Puri, R.K.; Basuki, I.; van Heist, M.; Wan, M.; Liswanti, N.; Rukmiyati; Sardjono, M.A.; Samsoedin, I. Exploring Biological Diversity, Environment and Local People's Perspectives in Forest Landscapes, 2nd ed.; CIFOR: Bogor, Indonesia, 2003. Available online: http://www.cifor.org/mla/_ref/publications/detail/exploring_biological_diversity.htm (accessed on 2 September 2015).

25. Sheil, D.; Puri, R.K.; Wan, M.; Basuki, I.; van Heist, M.; Liswanti, N.; Rukmiyati; Rachmatika, I.; Samsoedin, I. Recognizing local people's priorities for tropical forest biodiversity. Ambio 2006, 35, $17-24$.

26. Bemei, Y.; Watopa, Y. Tantangan di bentang alam mamberamo raya. Trop. Indone. 2006, 10, 14-16.

27. Boissière, M.; Liswanti, N.; Padmanaba, M.; Sheil, D. People Priorities and Perceptions. In Towards Conservation Partnership in Mamberamo; CIFOR: Bogor, Indonesia, 2007. Available online: http://www.cifor.org/mla/download/publication/People\%20priorities.pdf (accessed on 2 September 2015)

28. Supriatna, J.; Fretes, Y.; de Mack, A.; Yeager, C.P.; Olivieri, S.; Burnett, J.B.; Wijayanto, I.; Suryadi, S.; Suhandi, A. The Irian Jaya Biodiversity Conservation Priority-Setting Workshop (Final Report); Conservation International: Washington, DC, USA, 1999.

29. Diamond, J.M. Rediscovery of the Yellowfronted Gardener Bowerbird. Science 1982, 216, 431-434.

30. Diamond, J.M. New distributional records and taxa from the outlying mountain ranges of New Guinea. Emu 1985, 85, 65-91.

31. Boissière, M.; van Heist, M.; Sheil, D.; Basuki, I.; Frazier, S.; Ginting, U.; Wan, M.; Hariadi, B.; Hariyadi, H.; Kristianto, H.D.; et al. Pentingnya sumberdaya alam bagi masyarakat lokal di daerah aliran Sungai Mamberamo, Papua, dan implikasinya bagi konservasi. J. Trop. Ethnobiol. 2006, 1, 76-95.

32. Marshall, A.J.; Beehler, B.M. The Ecology of Papua, 1st ed.; Periplus Editions: Singapore, 2007.

33. Richards, S.J.; Suryadi, S. A biodiversity assessment of Yongsu-Cyclops mountains and the southern Mamberamo basin, Papua, Indonesia. In RAP Bulletin of Biological Assessment, 25; Conservation International: Washington, DC, USA, 2002.

34. Franky, Y.L.; Morgan, S. Papua Oil Palm Atlas: The Companies Behind the Plantation Explosion; Yayasan PUSAKA: Jakarta, Indonesia, 2015.

35. Van Heist, M.; Sheil, D.; Rachman, I.; Gusbager, P.; Raweyai, C.; Yoteni, H. The forests and related vegetation of Kwerba, on the Foja Foothills, Mamberamo, Papua (Indonesian New Guinea). Blumea J. Plant Taxon. Plant Geogr. 2010, 55, 153-161.

36. Sheil, D.; Liswanti, N. Scoring the importance of tropical forest landscapes with local people: Patterns and insights. Environ. Manag. 2006, 38, 126-136. 
37. Colfer, C.J.P.; Brocklesby, M.A.; Diaw, C.; Etuge, P.; Günter, M.; Harwell, E.; McDougall, C.; Porro, N.M.; Porro, R.; Prabhu, R.; et al. The Grab Bag: Supplementary Methods for Assessing Human Well-Being; The Criteria \& Indicators Toolbox Series, Number 6; CIFOR: Bogor, Indonesia, 1999. Available online: http://www.cifor.org/livesinforests/publications/pdf_files/toolbox-6c.pdf (accessed on 2 September 2015).

38. Maner, A. (Kwerba, West Papua, Indonesia). Personal communication, 2004.

39. Frazier, S. Crocodile Monitoring in Irian Jaya, Indonesia (Final Consultancy Report on FAO Project GCP/INS/060/JPN “Crocodile Industry Development on a Sustainable Basis”); Food and Agriculture Organization of the United Nations: Rome, Italy, 1989. Available online: http://agris.fao.org/aos/records/XF9091655 (accessed on 2 September 2015).

40. Cox, J. Development of the Crocodile Industry on a Sustainable Basis (Terminal Report FAO-PHPA Project GCP/INS/060/JPN); Food and Agriculture Organization of the United Nations: Rome, Italy, 1992. Available online: http://agris.fao.org/aos/records/XF9661333 (accessed on 2 September 2015).

41. Untung, B. (Natural Resource Conservation Agency or BKSDA, Jayapura, West Papua, Indonesia). Personal communication, 2004.

42. Sheil, D. Villagers: Villains or allies in forest biodiversity conservation? The IUCN/WWF Forest Conservation Newsletter. Arborvitae 2006, 30, 12.

43. Sheil, D.; Wunder, S. The value of tropical forest to local communities: Complications, caveats and cautions. Conserv. Ecol. 2002, 6, 9.

44. Redford, K.H.; Stearman, A.M. Forest-dwelling native Amazonians and the conservation of biodiversity. Conserv. Biol. 1993, 7, 248-255.

45. Lawrence, A.; Sheil, D. Tropical biologists, local people and conservation: New opportunities for collaboration. Trends Ecol. Evol. 2004, 19, 634-638.

46. Gadgil, M.; Rao, P.R.S.; Utkarsh, G.; Pramod P.; Ashwini Chhatre and Members of the People's Biodiversity Initiative. New meaning for old knowledge: The people's biodiversity registers program. Ecol. Appl. 2000, 10, 1307-1317.

47. Adams, M.; Aveling, R.; Brockington, D.; Dickson, B.; Elliot, J.; Hutton, J.; Roe, D.; Vira, B.; Wolmer, W. Biodiversity conservation and the eradication of poverty. Science 2004, 306, 1146-1149.

48. Berkes, F. Rethinking community-based conservation. Conserv. Biol. 2004, 18, 621-630.

49. Sheil, D.; Boissière, M.; Beaudoin, G. Unseen sentinels: Local monitoring and control in conservation's blind-spots. Ecol. Soc. 2015, 20, 39.

50. Boissière, M.; Sassen, M.; Sheil, D.; van Heist, M.; de Jong, W.; Cunliffe, R.; Wan, M.; Padmanaba, M.; Liswnati, N.; Basuki, I.; et al. Researching local perspectives on biodiversity in tropical landscapes: Lessons from ten case studies. In Taking Stock of Nature: Participatory Biodiversity Assessment for Policy Planning and Practice, 1st ed.; Lawrence, A., Ed.; Cambridge University Press: Cambridge, UK, 2010; pp. 113-141.

51. Garden. New Plants Found in Indonesian “Lost World” (News); Royal Horticultural Society: London, UK, 2006.

52. Garden. Plant Discoveries: Palm Tree is A New Genus; Royal Horticultural Society: London, UK, 2006.

53. Cyranoski, D. Calls to conserve biodiversity hotspots. Nature 2006, 439, 774. 
54. Beehler, B.M.; Prawiradilaga, D.M.; de Fretes, Y.; Kemp, N. A new species of Smoky Honeyeater (Meliphagidae: Melipotes) from western New Guinea. Auk 2007, 124, 1000-1009.

55. Padmanaba, M.; Boissière, M.; Ermayanti, S.; Sumantri, H.; Achdiawan, R. Perspectives on Collaborative Land Use Planning in Mamberamo Raya Regency, Papua, Indonesia; Case studies from Burmeso, Kwerba, Metaweja, Papasena, and Yoke; Project Report; CIFOR: Bogor, Indonesia, 2012. Available online: http://www.cifor.org/mla/download/publication/ Mamberamo_en_web.pdf (accessed on 2 September 2015).

56. Somba, N.D. Mamberamo Raya strives to conserve forested lands. The Jakarta Post. Available online: http://www.thejakartapost.com/news/2012/05/29/mamberamo-raya-strives-conserveforested-lands.html (accessed on 2 September 2015).

57. Indopacific Conservation Alliance IPAC. Facilitating community-driven conservation and strengthening local cultural institutions in Asmat and the Greater Lorentz Lowlands, 2012. Available online: http://indopacific.org/asmat.asp (accessed on 2 September 2015).

58. Agrawal, A.; Angelsen, A. Using community forest management to achieve REDD+ goals. In Realising REDD+. National Strategy and Policy Options; Angelsen, A., Ed.; CIFOR: Bogor, Indonesia, 2009.

59. Ostrom, E.; Burger, J.; Field, C.B.; Norgaard, R.B.; Policansky, D. Revisiting the commons: Local lessons, global challenges. Science 1999, 284, 278-282.

60. Cole, D.; Ostrom, E. The Variety of Property Systems and Rights in Natural Resources; Environmental Affairs Research Paper No. 2010-08-01; Indiana University-Bloomington School of Public and Environmental Affairs: Bloomington, IN, USA, 2010.

61. FAO. Tenure Security for Better Forestry. Understanding Forest Tenure in South and Southeast Asia; Food and Agriculture Organization of the United Nations: Rome, Italy, 2007.

62. Porter-Bolland, L.; Ellis, E.A.; Guariguata, M.R.; Ruiz-Mallén, I.; Negrete-Yankelevich, S.; Reyes-García, V. Community managed forests and forest protected areas: An assessment of their conservation effectiveness across the tropics. For. Ecol. Manag. 2012, 268, 6-17.

(C) 2015 by the authors; licensee MDPI, Basel, Switzerland. This article is an open access article distributed under the terms and conditions of the Creative Commons Attribution license (http://creativecommons.org/licenses/by/4.0/). 